\title{
VLBI astrometry for the NASA/Stanford gyroscope relativity mission Gravity Probe B
}

\author{
N. Bartel ${ }^{1}$, R. R. Ransom ${ }^{1 *}$, M. F. Bietenholz ${ }^{1}$, D. E. Lebach ${ }^{2}$, \\ M. I. Ratner ${ }^{2}$, I. I. Shapiro ${ }^{2}$ and J.-F. Lestrade ${ }^{3}$ \\ ${ }^{1}$ York University, Toronto, ONT, Canada, \\ ${ }^{2}$ Harvard-Smithsonian Center for Astrophysics, Cambridge, MA., U. S. A. \\ ${ }^{3}$ Observatoire de Paris, Paris, France \\ *now at DRAO, Penticton, Canada
}

\begin{abstract}
We used VLBI observations at $8.4 \mathrm{GHz}$ between 1991 and 2005 to determine the motion of the RS CVn binary IM Pegasi (HR 8703), the guide star for the NASA/Stanford gyroscope relativity mission, Gravity Probe B (GP-B). The motion was determined relative to our primary reference, the core of the quasar 3C 454.3. The stability of this core was checked relative to two other extragalactic sources, B2250+194 and B2252+172, the former of which was tied to the ICRF. The core of $3 \mathrm{C} 454.3$ is stationary relative to these two sources to within $30 \mu$ as $\mathrm{yr}^{-1}$ in each coordinate. IM Pegasi's radio morphology varies, but appears to be on average centered on the primary. We estimate the proper motion of IM Pegasi with a statistical

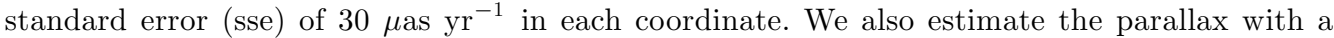
statistical standard error of $75 \mu$ as and parameters of the orbit with sse's corresponding to 110 $\mu$ as on the sky. Coupled with our upper limit of three times the sse on any systematic errors in each parameter estimate, these results ensure that the uncertainty of IM Pegasi's proper motion makes only a small contribution to the uncertainty of GP-B's tests of general relativity.
\end{abstract}

Keywords. relativity, techniques: interferometric, reference systems, stars: imaging, stars: distances, radio continuum: stars

\section{Introduction}

Gravity Probe B (GP-B) is the spaceborne relativity experiment developed by NASA and Stanford University to test two predictions of general relativity (GR) with gyroscopes in low-earth orbit. GP-B sought to measure the precession of the gyroscopes relative to distant inertial space, represented by an extragalactic reference frame. Because of technical restrictions, the spacecraft could not measure the precession directly relative to that frame but only to an optically bright star, the guide star chosen to be the RS CVn binary IM Pegasi (HR 8703). We determined IM Pegasi's motion independently relative to the extragalactic reference frame. In particular, we used VLBI to determine this motion relative to the core of the quasar 3C 454.3 which we tied to two other extragalactic sources. The motion of one of them was measured directly within the International Celestial Reference Frame (ICRF).

\section{Observations and results}

Between 1997 and 2005, we carried out 35 sessions of VLBI observations at $8.4 \mathrm{GHz}$ of IM Pegasi and two extragalactic sources, 3C 454.3 and B2250+194, supplemented by another (likely) extragalactic source, B2252+172 for the last third of the sessions. We used 
a worldwide network of radio telescopes including the 10 dishes of the Very Long Baseline Array (VLBA) and the Very Large Array of the National Radio Astronomy Observatory in the USA, the Effelsberg telescope of the Max-Planck-Institut für Radiostronomie in Germany, and the 70-m dishes of the NASA Deep Space Network in the USA, Spain, and Australia. Each session used typically 15 telescopes and was of about 15 hours in length. The data were correlated using the VLBA processor in Socorro, NM, USA. We also used results from 1991-1994 observations of IM Pegasi and 3C 454.3.

We located three major components in 3C 454.3 by fitting Gaussians to the brightness distributions. The most easterly of these components is the most stable, and stationary

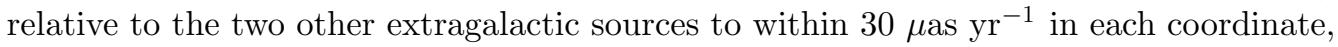
corresponding to a limit of $0.75 \mathrm{c}$ in each coordinate for a flat universe with $H_{0}=70$ $\mathrm{km} \mathrm{s}^{-1} \mathrm{Mpc}^{-1}, \Omega_{M}=0.3$, and $\Omega_{\lambda}=0.7$. This component is most likely closely related to the core and the gravitational center of the quasar and is used by us as the primary reference for IM Pegasi. The radio morphology of IM Pegasi varies, having sometimes one and sometimes two components (Figure 1), and at one epoch an even more complex structure. The radio emission is thought to originate in flares and magnetic loops above the surface of the star. Our astrometry shows that the radio emission is centered close to the primary on the plane of the sky (Figure 1).

We fit a 9-parameter model to the position of the centroid of the radio images of IM Pegasi at all 39 epochs; our estimate of the proper motion has a statistical standard error (sse) of $30 \mu \mathrm{as} \mathrm{yr}^{-1}$ in each coordinate. In addition we estimate the parallax with an sse of $75 \mu$ as and the parameters of the orbit with sse's corresponding to $110 \mu$ as on the sky. The systematic errors for each of these estimates are almost surely no more than threefold higher than the sse's. The release of our other astrometric results will be coordinated with the NASA/Stanford GP-B team. Our VLBI astrometry program surpassed its accuracy goal, thereby ensuring that the uncertainty of IM Pegasi's proper motion will make only a small contribution to the uncertainty of the GP-B tests of general relativity.

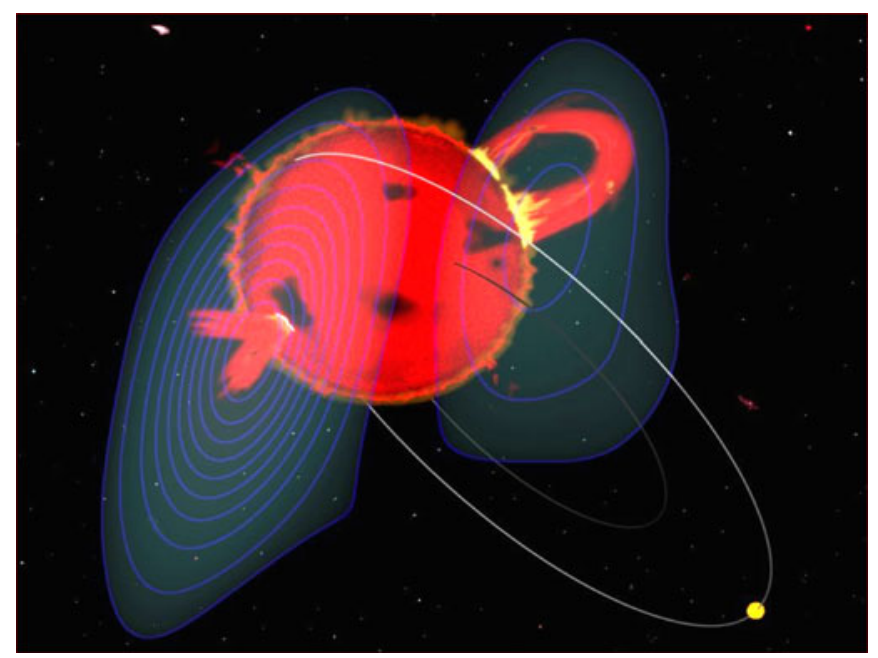

Figure 1. An artist's impression of IM Pegasi, showing the K2 primary, the much smaller secondary in the lower right, and their orbits. The radio emission seems to originate near the primary. Two flares represent the possible source of the radio emission. Superposed are contours representing the radio emission for epoch 1999 September 18/19. Two radio components appeared at this epoch. Contours are at $5,10,20,30, \ldots, 80$, and $90 \%$ of the peak brightness of $11.5 \mathrm{mJy} /$ beam. The scale and orientation of this figure will be released later (see text). 